\title{
АЛГОРИТМ УДЕРЖАНИЯ КУРСА БПЛА ПУТЁМ ПЕРЕРАСПРЕДЕЛЕНИЯ УПРАВЛЯЮЩИХ СИГНАЛОВ
}

\section{ALGORITHM FOR HOLDING THE UAV COURSE BY REDISTRIBUTING CONTROL SIGNALS}

\section{N. Nersisyan}

Summary. For multicopters that are UAVs with three or more rotors, the output control signal is a PWM (Pulse Width Modulation) signal, which determines the value of the angular velocity of a given motor. The purpose of the article is show that redistributing the output PWM signals with a certain function depending on the value of the yaw angle, the UAV will maintain its original direction, thus ignoring the rotation around its own axis.

As a result of the given equations, the angular movements of the UAV can be observed with respect to a stationary coordinate system. The task is to bring a new algorithm for keeping the course of an unmanned aerial vehicle (UAV) when the yaw angle changes.

The given functions are periodic; they transfer the PWM value of the given motor; therefore, the value of the angular velocity to the next motor. The transferred value of depends on the size of the yaw angle.

The algorithm simplifies the control of UAVs, as the yaw angle is ignored, and it is sufficient to set only the desired pitch and roll angles or angular velocities. As a result, the algorithm can be integrated into the UAV control system as a separate block, with the control PWM signals at the input, and the new PWM values at the output, which are given to the motor speed controllers.

Keywords: unmanned aerial vehicle, quadcopter, algorithm, redistribution, direction, yaw, pwm.

\author{
Нерсисян Нерсес Грачевич \\ Начиональный Политехнический Университет \\ Армении \\ nerses98@mail.ru
}

Аннотация. Для мультикоптеров которые являются БПЛА с тремя и более несущими винтами, выходным управляющим сигналом является сигнал ШИМ (широтно-импульсная модуляция), который задаёт величину угловой скорости конкретного мотора. Цель статьи является показать, что в зависимости от значения угла рыскания путем перераспределения выходных ШИМ сигналов определённой функцией, мультикоптер будет сохранить свой исходный курс, таковым пренебрегая поворот вокруг своей оси. С помощью приведенных уравнений можно рассмотреть движения летательного аппарата относительно неподвижной системой координат. Задачей является приведение нового алгоритма для удержания курса беспилотного летательного аппарата (БПЛА), при изменении угла рыскания.

Приведенные функции являются периодичными и передают значение ШИМ, следовательно, и значение угловой скорости конкретного мотора к последующему ему мотору, а величина переданного значения зависит от величины угла рыскания.

Алгоритм облегчает управления БПЛА, так как для управления в любом направлении пренебрегается угол рыскания и достаточно задать только желаемые углы или угловые скорости тангажа и крена. В результате приведённый алгоритм можно интегрировать В систему управления БПЛА, как отдельный блок, которому на вход подаются управляющие ШИМ сигналы, а в выходе новые значения ШИМ сигналов подаются на вход регуляторов скорости моторов.

Ключевые слова: беспилотный летательный аппарат, квадрокоптер, алгоритм, перераспределение, курс, рыскание, шим.

нанесение ударов на важную технику: так называемые камикадзе дроны, последним оснащают взрывателями.

Из перечини разных видов бпла широко используются мультикоптеры, которые бывают оснащены тремя или более моторами. На рис. 1 показан пример шести винтового мультикоптера гексакоптера.

У мультикоптеров каждый пропеллер обладает своим двигателем. Например, у четырехвинтового коптера квадрокоптера, существуют соответственно четыре дви- 


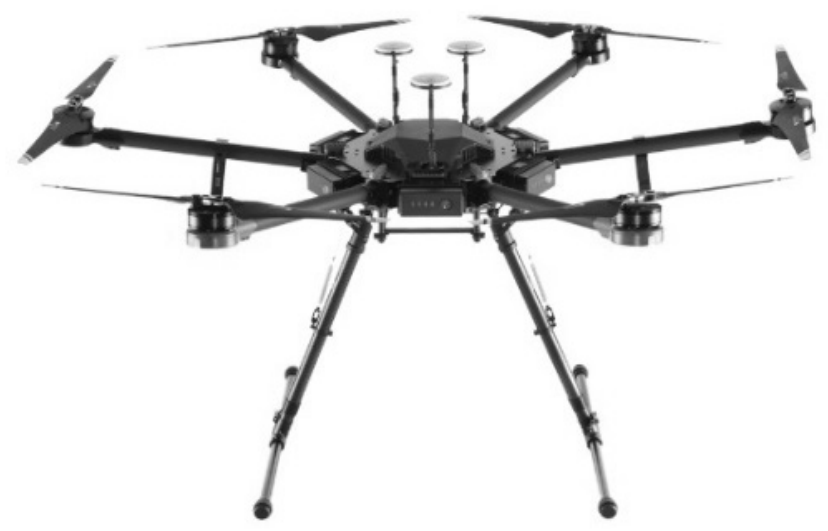

Рис. 1 Мультикоптер с шестью моторами

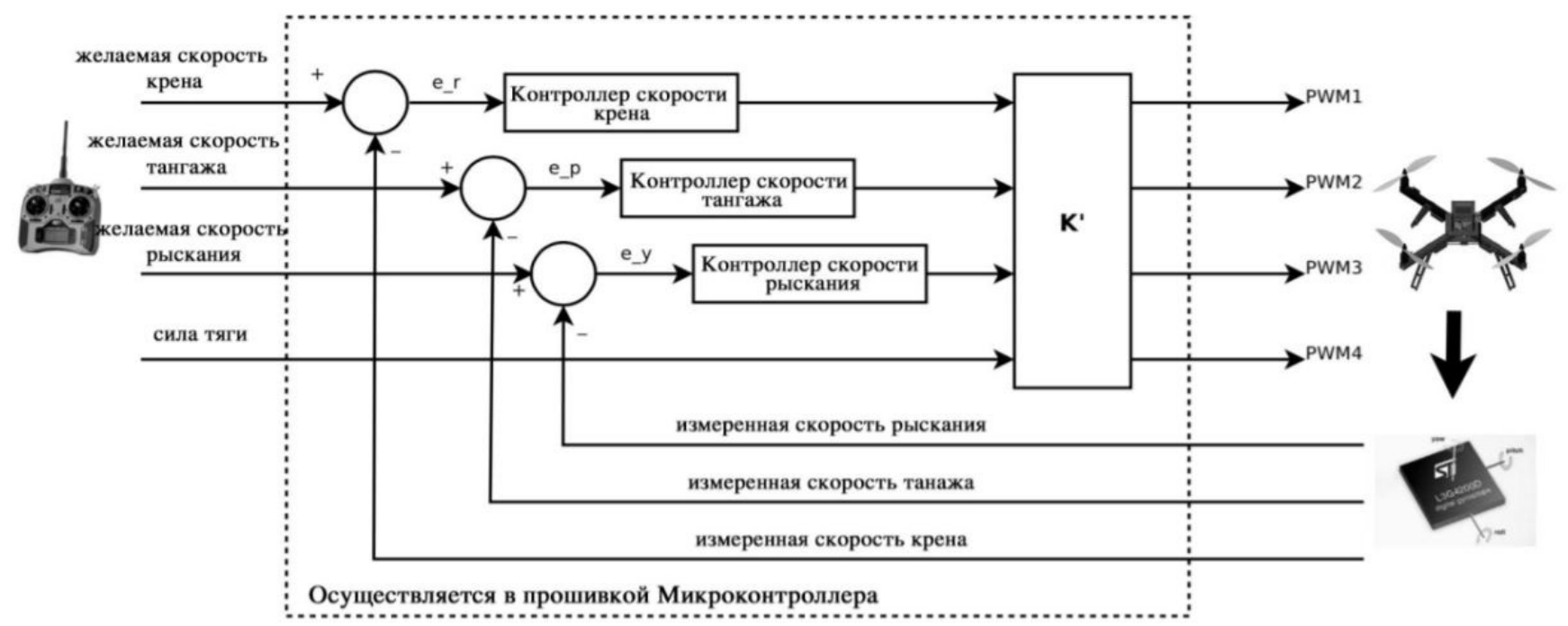

Рис. 2 Схема системы управления квадрокоптера

гателя с собственными аккумуляторами. Пара винтов осуществляет вращение против часовой стрелки, а другая пара вращение по часовой стрелки, следовательно, необходимость в хвостовом винте отсутствует.

Так как мультикоптеры можно эксплуатировать в таких местах и условиях, где человеку может грозить опасность, для успешного выполнения подобных операций и по мере распространения использования мультикоптеров, перед ними ставятся более трудные задачи, на пример в вышеперечисленных ситуациях возникают дополнительные сложности, связанные с обеспечением довольно устойчивого полёта, с системой управления, с точностью и правильной эксплуатацией летательного аппарата.

Схема управления квадрокоптером. На рисунке 2 показана общая схема управления на примере квадрокоптера, который оснащен четырьмя бесщёточными моторами [1].
Как видно из рис. 2 основой управления является задающие сигналы управления в этом случае желаемая скорость крена $\dot{\phi}$, желаемая скорость тангажа $\dot{\theta}$, рыскания $\dot{\psi}$ и сила тяги $F$. Эти управляющие сигналы или желаемые значения задаются с помощью пульта дистанционного управления или автопилота в зависимости от режима полёта. На квадрокоптере установлен вычислительное устройство автопилот, к которому подключены датчики, такие как инерциальный модуль (IMU) включающий в себе гироскоп, акселерометр, магнитометр и барометр для считывания высоты с помощью изменения давления. Инерциальные датчики измеряют углы отклонения или угли Эйлера или их скорости по трем осям, давая данные автопилоту про угловое расположение или угловую скорость квадрокоптера в пространстве.

Как показано на рис. 2, разница между желаемыми и измеренными значениями $\left(e_{r}, e_{p}, e_{y}\right)$ подаётся на вход 


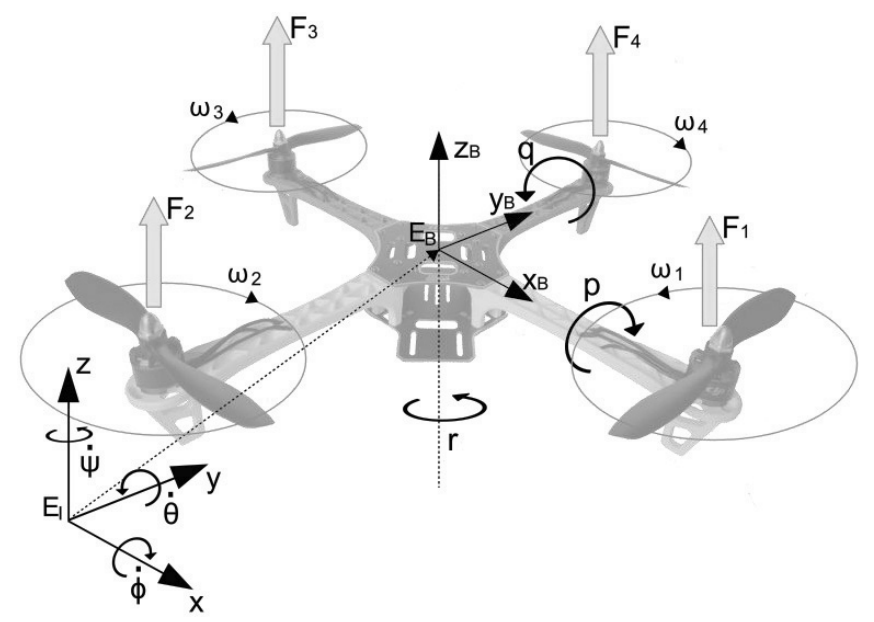

Рис. 3. Схематическое представление квадрокоптера

$$
R=\left[\begin{array}{ccc}
\cos \psi \cos \theta-\sin \phi \sin \psi \sin \theta & -\cos \phi \sin \psi & \cos \psi \sin \theta+\cos \theta \sin \phi \sin \psi \\
\cos \theta \sin \psi+\cos \psi \sin \phi \sin \theta & \cos \phi \cos \psi & \sin \psi \sin \theta-\cos \psi \cos \theta \sin \phi \\
-\cos \phi \sin \theta & \sin \phi & \cos \phi \cos \theta
\end{array}\right]
$$

\begin{tabular}{ll}
\hline$F_{i}=c_{M} \Omega_{i}^{2}$ & $(2)$ \\
\hline$\phi_{1}=f(\psi, \phi)$ & $(3)$ \\
\hline$\theta_{1}=f(\psi, \theta)$ & $(4)$ \\
\hline$\left\{\begin{array}{l}\Omega_{1}^{\prime}=f_{1}\left(\psi, \Omega_{1}\right) \\
\Omega_{2}^{\prime}=f_{1}\left(\psi, \Omega_{2}\right) \\
\Omega_{3}^{\prime}=f_{1}\left(\psi, \Omega_{3}\right) \\
\Omega_{4}^{\prime}=f_{1}\left(\psi, \Omega_{4}\right)\end{array}\right.$
\end{tabular}

регулятору который обозначен $K^{\prime}$. Регулятор - это математическое устройство, алгоритм, задача которого минимизировать $e_{r}, e_{p}, e_{y}$, таким образом обеспечив чтобы угловые скорости и тяги всегда стремились к желанным значениям. На выходе регулятора получаются управляющие ШИМ сигналы, которые уже подаются на электронные регуляторы скорости оборотов моторов (ESC). Управляющие ШИМ сигналы являются конечными значениями в системе управления. ШИМ сигнал можно прямо-пропорционально сопоставить с угловой скоростью конкретного мотора.

На рис. $3 E_{I}$ обозначена инерциальная или неподвижная система координат с осями $x_{I}, y_{I}, z_{I}$, а $O_{B}$ система координат, связанная с корпусом квадрокоптера с осями $x_{B}, y_{B}, z_{B}$. Ориентация корпуса квадрокоптера относительно инерциальной системы координат описывается ортогональной матрицей поворота $R$ [2] (формула 1).

Назначим значения угловых скоростей моторов соответственно $\Omega_{1}, \Omega_{2}, \Omega_{3}, \Omega_{4}$ (рис. 3). Известно, что пропел- лер каждого мотора создаёт тягу $F_{i}$ который пропорционален квадрату угловой скорости $i$-го мотора $\Omega_{i}$,

где $c_{M}=$ const.

\section{Описание залачи и приведение алгоритма}

На рис. 3 видно, что при вращении летательного аппарата вокруг оси $z_{b}$ вместе с ним вращаются и связанные с ним оси $x_{B}$ и $y_{B}$. То есть относительно неподвижной системы координат $x_{I}, y_{I}, z_{I}$, оси $x_{B}$ и $y_{B}$ вращаются ровно на угол $\psi$ [3]. Из-за этого при управлении летательным аппаратом в определённом направлении, если до поворота оператор задавал желаемые значения углов крена и тангажа $\varphi$ и $\theta$, то после поворота на некоторый угол $\psi$, если необходимо лететь в том же направлении относительно неподвижной системы координат $O_{I}$, то необходимо задавать новые значения углов $\varphi_{1}$ и $\theta_{1}$ которые связаны с $\varphi$ и $\theta$ некоторой функцией от $\psi$. 


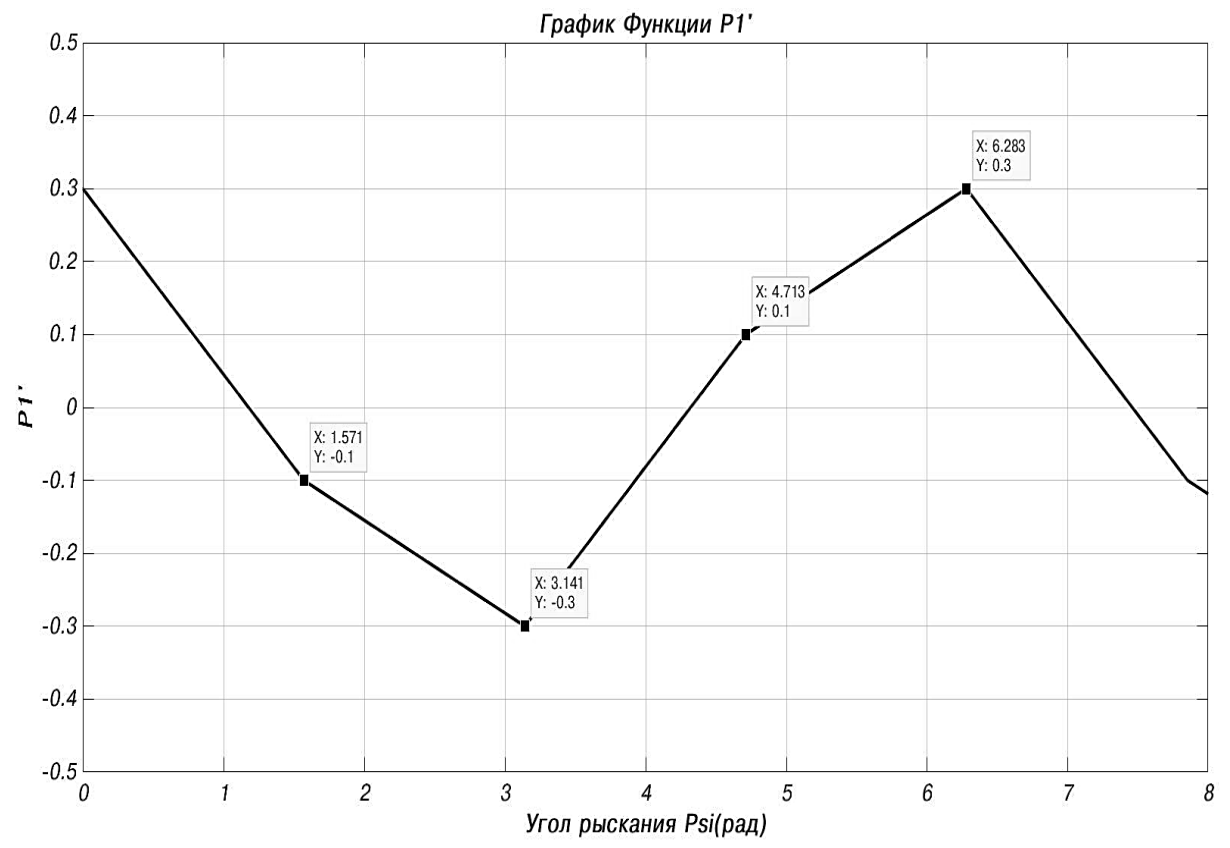

Рис. 4. Графическое представление функции (8)-го уравнения

$P_{1}^{\prime}=\frac{2}{\pi} *(\arcsin (\cos (\psi)) * P 1+\arcsin (\sin (\psi)) * P 2)$

$P_{2}^{\prime}=\frac{2}{\pi} *(\arcsin (\cos (\psi)) * P 2+\arcsin (\sin (\psi)) * P 3)$

$P_{3}^{\prime}=\frac{2}{\pi} *(\arcsin (\cos (\psi)) * P 3+\arcsin (\sin (\psi)) * P 4)$

$P_{4}^{\prime}=\frac{2}{\pi} *(\arcsin (\cos (\psi)) * P 4+\arcsin (\sin (\psi)) * P 1)$

$\left[\begin{array}{l}P_{1}^{\prime} \\ P_{2}^{\prime} \\ P_{3}^{\prime} \\ P_{4}^{\prime}\end{array}\right]=\frac{2}{\pi} *\left[\begin{array}{ll}P_{1} & P_{2} \\ P_{2} & P_{3} \\ P_{3} & P_{4} \\ P_{4} & P_{1}\end{array}\right]\left[\begin{array}{l}\arcsin (\cos (\psi)) \\ \arcsin (\sin (\psi))\end{array}\right]$

$\left[\begin{array}{l}P W M_{1}^{\prime} \\ P W M_{2}^{\prime} \\ P W M_{3}^{\prime} \\ P W M_{4}^{\prime}\end{array}\right]=\left[\begin{array}{l}P_{1}^{\prime} \\ P_{2}^{\prime} \\ P_{3}^{\prime} \\ P_{4}^{\prime}\end{array}\right]+P W M 0$ 


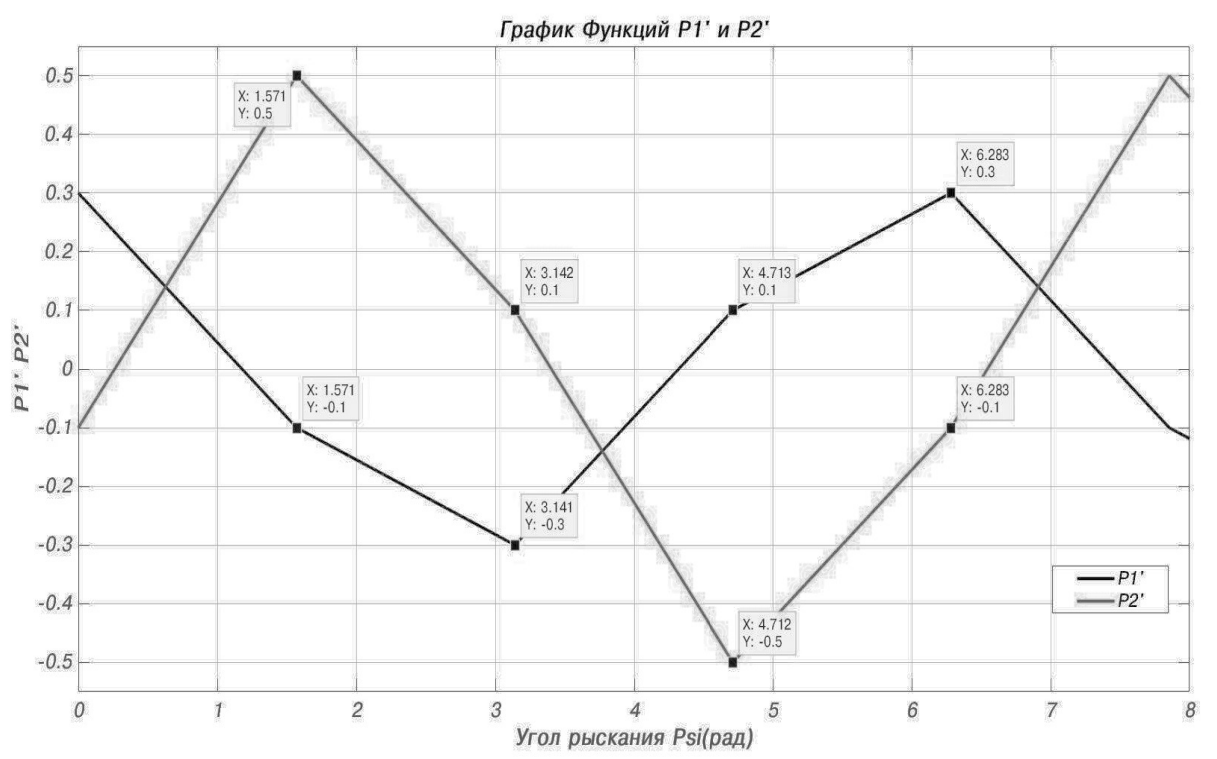

Рис. 5 Графическое представление функций (8)-го и (9)-го уравнения

В случаях дальних полётов или автоматических миссиях это вызывает дополнительные сложности управления, таковым обязуя оператора следить за углом рыскания.

В уравнениях (3) и (4) углы $\theta, \varphi$, связаны с тягами уравнением (2), а те пропорционально связаны с угловыми скоростями $\Omega_{1}, \Omega_{2}, \Omega_{3}, \Omega_{4}$ как показано в (2). Получается, что после поворота на угол $\psi$ и задавания новых значений $\theta_{1}$ и $\varphi_{1}$, угловые скорости моторов тоже примут новые значения $\Omega^{\prime}{ }_{1}, \Omega^{\prime}{ }_{2}, \Omega^{\prime}{ }_{3}, \Omega^{\prime}{ }_{4}$. Получится система уравнений (5)

В (5) также один из двух аргументов угол рыскания $\psi$.

Известно, что угловые скорости $\Omega, \Omega_{2}, \Omega_{3}, \Omega_{4}$ прямо пропорционально связаны с значениями скважности (Duty Cycle) управляющих ШИМ сигналов [4]. Получается уравнение (5) можно переписать для ШИМ сигналов. Назначим $P W M 0$ значение ШИМ сигнала, которое одинаковое для четырёх моторов. $P W M 0$ это значение необходимое для парения в воздухе. Для упрощения допустим $P W M 0=$ const. Результирующий ШИМ сигнал должен иметь вид $P W M_{i}=P_{i}+P W M 0$ где $i=1,2,3,4$ для квадрокоптера. $P_{i}$ принимает значения в диапазоне от $-k \leq P_{i} \leq k$.

Так как $P W M 0=$ const, то для достижения перераспределения ШИМ сигналов можно перераспределять только значения $P_{i}$ а потом прибавить $P W M 0$ После перераспределения значений $P_{i}$, полученные новые значения назначим $P_{i}^{\prime} i=1,2,3,4$. В конце итоговый
ШИМ сигнал должен иметь вид $P W M_{i}^{\prime}=P_{i}^{\prime}+P W M 0$ . В итоге функция перераспределения имеет следующий вид.

В уравнениях (6)-(9) присутствуют функции $y=\arcsin (\cos (x))$ и $y_{1}=\arcsin (\sin (x))$ которые по отдельности $2 \pi$ периодичные функции.

Отдельно рассмотрим уравнение (6), при случае $\psi=0^{\circ}, P_{1}^{\prime}=P 1$. При $\psi=90^{\circ} P_{1}^{\prime}=P 2$, при $\psi=180^{\circ} P_{1}^{\prime}=-P 1$. Получается отдельно взятое (6) уравнение при повороте угла рыскания от 0 до 360 градусов изменяет значение $P^{\prime}{ }_{1}$ периодичным образом так, что в точках $0^{\circ} \rightarrow 90^{\circ} \rightarrow 180^{\circ} \rightarrow 270^{\circ} \rightarrow 360^{\circ}$ она принимает соответственно значения $P 1 \rightarrow P 2 \rightarrow-P 1 \rightarrow-2 \rightarrow P 1$. После перераспределения значений добавляя к каждому значению константу $P W M 0$, получатся новые значения управляющих ШИМ сигналов. На пример допустим $P W M 0=1.5$ мc a $k=0.5$, для $p 1=0.3$ и $P W M 0=-0.1$ мc график функции в (6) уравнении

$$
\begin{aligned}
& f(x)=\frac{2}{\pi} *(\arcsin (\cos (\psi)) * 0.3+ \\
& +\arcsin (\sin (\psi)) *(-0.1))
\end{aligned}
$$

имеет следующий вид.

Как видно из рис. 4, значение $P_{1}^{\prime}$ по мере изменения аргумента, угла $\psi$, переходит из значения $P 1$ в точке $\psi=$ 0 рад к $P 2$ в точке

$$
\psi=\frac{\pi}{2} \text { рад }
$$


а в точках $\psi=\pi$ рад и $\psi=\frac{2 \pi}{3}$ рад

уже переходит к $-P 1$ и $-P 2$ соответственно.

Допустим $P 3=0.5$ мc. Графические представления функций $P_{1}^{\prime}$ и $P_{2}^{\prime}$ вместе будут иметь следующий вид (рис. 5).

Из рис. 5 видно, что в точке

$\psi=\frac{\pi}{2} P_{1}^{\prime}$

принимает значение $P 2$ а $P_{2}^{\prime}$ принимает значение $P 3$.

Если рассмотреть всю систему уравнений (6) - (9), то на пример при угле рыскания $\psi=90^{\circ}$ новые значения $P_{i}$ будут следующими. $P_{1}^{\prime}=P 2, P_{2}^{\prime}=P 3, P_{3}^{\prime}=P 4$, $P_{4}^{\prime}=P 1$.

Получается система уравнений (6)-(9) перераспределяет компонент ШИМ сигнала $P_{i}$ в зависимости от значения угла рыскания $\psi$, таким образом что относительно неподвижной системы координат $E_{I}$ летательный аппарат будет лететь в том же направлении не смотря на поворот вокруг своей оси $z_{B}$.

Представим уравнения (6)-(9) в матричном виде, где $i$ $=1,2,3,4$ (формула 10).

Где число строк равно количеству моторов соответственно и ШИМ сигналов.
Исходя из задачи что выходное значение в уравнениях (13) $\mu s_{i}^{\prime}$ не может превосходить исходные значения $P_{i}$ и $P s_{i}+1 i=1,2,3,4$, поэтому были рассмотрены функции $\arcsin (\cos (x))$ и $\arcsin (\sin (x))$.

В конце итоговый ШИМ сигнал будет следующим (формула 11).

Если в уравнении (13) в аргументе к углу рыскания $\psi$ прибавить значение углового отклонения носа БПЛА от севера, то получится что БПЛА будет всегда лететь в направлении к северу или югу осью тангажа, и к западу или востоку осью крена.

Можно заключить что полученная система уравнений (11) переставляет или перераспределяет последующие выходные для системы управления ШИМ сигналы в зависимости от угла рыскания таким образом, что полученные новые значения сигналов заставляют БПЛА поддержать прежний курс не смотря на поворот во круг своей оси $z_{B}$. Таковым получается, что мы пренебрегаем третью ось $z_{B}$ для угловых изменений и можем рассмотреть движения летательного аппарата относительно неподвижной системой координат. Этот алгоритм применим также и для гексакоптеров и для октокоптеров, и для летательных аппаратов с произвольным калечеством моторов и облегчает управления БПЛА для учебных целей, а также в сложных миссиях, где невозможно следить за курсом БПЛА.

\section{ЛИТЕРАТУРА}

1. Kyaw Myat Thua, A.I. Gavrilova // Designing and modeling of quadcopter control system using L1 adaptive control, Procedia Computer Science Volume 103, 2017, Pages 528-535.

2. О.Н. Гаспарян, Г.Г. Егиазарян, А.Г. Дарбинян, Д.У. Егиазарян // Разработка системы управления квадрокоптером с развязывающим регулятором с учетом динамики электродвигателей — Вестник российско-армянского (славянского) университета: физико-математические и естественные науки- 2017, стр-59-80

3. Бессекерский В.А., Попов Е.П. Теория систем автоматического регулирования.-М.: Наука, 2003.

4. А.В. Давтян, Н.Г. Нерсисян, О.Н. Гаспарян // Система управления БЛА и выбор коэффициентов, пропорционального интегрально-дифференциального регулятора в программной среде SIMULINK 2019, стр. 17-18 